\title{
MODELAGEM DA RELAÇÃO HIPSOMÉTRICA EM PLANTIOS DE JATOBÁ (Hymenaea courbaril L.) NA AMAZÔNIA CENTRAL
}

\author{
MODELING HEIGHT-DIAMETER RELATIONSHIP IN JATOBÁ (Hymenaea courbaril L.) \\ STANDS IN THE CENTRAL AMAZON
}

\author{
Filipe Campos de Freitas ${ }^{1}$, Juliana Torres Silva', Nabor da Silveira Pio ${ }^{3}$, \\ Roberval Monteiro Bezerra de Lima ${ }^{4}$ \\ ${ }^{1}$ Universidade Federal de Lavras, Lavras, Minas Gerais, Brasil, - filipecamposdefreitas@hotmail.com \\ ${ }^{2}$ Greenpeace Brasil, Manaus, Amazonas, Brasi,_juli_atm87@hotmail.com \\ ${ }^{3}$ Universidade Federal do Amazonas, Manaus, Amazonas, Brasil-nspio.ufam@gmail.com \\ ${ }^{4}$ Empresa Brasileira de Pesquisa Agropecuária, Manaus, Amazonas, Brasil - roberval.lima@embrapa.br
}

RESUMO

Com o intuito de valorizar espécies amazônicas provenientes de plantios, o objetivo deste estudo foi avaliar o ajuste de modelos hipsométricos para altura individual total e comercial de árvores de Jatobá em plantios homogêneos com idades de 17 e 24 anos. Foi realizado um inventário $100 \%$ nos dois plantios, sendo coletadas a circunferência à altura do peito (CAP), a altura total (HT) e a altura comercial $(\mathrm{HC})$ das árvores. Foram testados 16 modelos hipsométricos verificados na literatura especializada. Foram adotados como critérios de seleção do melhor modelo as seguintes variáveis: significância da estatística $F$ da Análise de Variância (ANOVA) da regressão; coeficiente de determinação ajustado ( $R^{2}$ aj.); erro padrão da estimativa (Syx\%); análise gráfica dos resíduos. Foi realizado o teste Qui-Quadrado para avaliar se os valores estimados foram iguais aos valores observados. Uma maior homogeneidade de dados foi encontrada para o plantio de 17 anos. Todos os ajustes apresentaram significância ao nível de $1 \%$ de probabilidade. As melhores estimativas foram verificadas para altura total no plantio de 17 anos. Foi observada diferença significativa entre os valores estimados e observados de altura total para o plantio de 24 anos. O modelo hiperbólico foi o mais apropriado para estimativas tanto de altura total quanto de altura comercial.

PALAVRAS-CHAVE: Altura, Plantios comerciais, Recuperação de áreas degradadas.

\section{ABSTRACT}

In order to value Amazonian species from plantations, the objective of this study was to evaluate the fit of hypsometric models for total and commercial individual height of Jatobá trees in 17 and 24 years old homogeneous stands. It was done a $100 \%$ forest inventory which it was measured the circumference at breast height (CAP), commercial height $(\mathrm{HC})$ and total height $(\mathrm{HT})$ of the trees. It was fitted 16 hypsometric models verified in the specialized literature. The following variables were adopted as criteria for selecting the best model: significance of $F$ statistics according regression's analysis of variance; adjusted determination coefficient $\left(R^{2}\right.$ aj.); standard error of estimate (Syx\%); graphic residual analysis. Chi-square test was performed to evaluate if the estimated values were equal to the observed values. The 17-years-old stand showed greater data homogeneity. All fitted models presented significance at the $1 \%$ probability level. Better estimates were found for total height from 17-year-old stand. Significant difference was observed between estimated and observed total height values for the 24-year-old stand. The hyperbolic model was the most appropriate for both estimates, total and commercial height.

KEYWORDS: Height, Comercial stand, Recovery of degraded areas. 


\section{INTRODUÇÃO}

O processo de degradação ou ocupação da terra na região amazônica, em geral, tem-se iniciado a partir da exploração madeireira, retirando-se árvores de alto valor comercial, seguida pela remoção da vegetação secundária pelo fogo, e posteriores usos agrícolas nas áreas desmatadas (KELLER et al., 2004; MONTEIRO et al., 2004; ALVES et al., 2009). A partir desse cenário, a preocupação com o uso sustentável dos recursos naturais existentes torna-se explícita, bem como a necessidade de recuperar e incorporar as áreas alteradas ao processo produtivo, sendo imprescindível portanto a adoção de medidas visando a ocupação mais racional da Amazônia e a ampliação do conhecimento sistematizado e científico da região, especificamente da pesquisa agropecuária e florestal (SOUZA et al., 2008).

Plantações florestais se mostram uma boa alternativa na reintegração das áreas alteradas ao processo produtivo, uma vez que podem contribuir significativamente com o aumento da oferta de madeiras de elevado valor econômico, diminuir a pressão sobre as florestas nativas, além da prestação de serviços ambientais. O reflorestamento, especialmente com árvores de uso múltiplo em monocultivo ou em sistemas agroflorestais, já é praticado e varia de acordo com as características ambientais e socioeconômicas de cada região (MIRANDA \& VALENTIM, 2000; BRIENZA JÚNIOR et al., 2008).

Neste sentido, o Jatobá (Hymenaea courbaril L.), uma das espécies mais populares da Amazônia, atende a várias características necessárias para a recuperação de áreas degradadas ou enriquecimento florestal. Segundo Lorenzi (2002), a espécie é pouco exigente em fertilidade e umidade do solo, de fácil multiplicação, pois produz anualmente grande quantidade de sementes viáveis que germinam rapidamente, sendo essencial na composição de reflorestamentos e arborização. Além da madeira utilizada na construção civil e laminação, a árvore possui resina e casca bastante valorizadas popularmente, além de frutos comestíveis (MELO et al., 2004; CAMPOS FILHO \& SARTORELLI, 2015).

O florestamento ou reflorestamento com espécies nativas possui papel claro na proteção dos recursos hídricos e edáficos, na recuperação de áreas degradadas e na expectativa futura de valorização dos produtos florestais devido a aceleração do desmatamento e restrições legais, sendo uma alternativa viável sob os pontos de vista ecológico, econômico e social na Amazônia (TONINI et al., 2005). O êxito na condução destes plantios passa pela utilização de técnicas que possibilitem a mensuração das variáveis dendrométricas necessárias para estimativas de volume e/ou biomassa com maior precisão possível. Para isso, se busca a mensuração de dimensões básicas como Diâmetro à Altura do Peito (DAP) e a altura de árvores (FELICIANO et al., 2016).

A medição da altura de uma árvore demanda mais tempo que uma simples medição de diâmetro com fita métrica, pois está sujeita a fatores que dificultam sua execução: dificuldade de observar o ápice da árvore, principalmente em povoamentos mais adensados; inclinação do terreno; falta de treinamento para correto manuseio dos equipamentos. Consequentemente, um maior tempo de inventário proporciona maiores custos (SANQUETTA et al., 2014; SILVA et al., 2017). Dessa forma, é procedimento comum na condução de plantios florestais a medição da altura de algumas árvores para o ajuste de modelos que expressem uma relação hipsométrica que gere estimativas da altura das árvores não medidas. Uma relação biométrica bastante empregada nestas estimativas é diâmetro-altura, com possibilidades de transformação destas variáveis de acordo com as características dos dados para melhor ajuste (MACHADO \& FIGUEIREDO FILHO, 2003; SCHNEIDER et al., 2009).

Na região amazônica, cada vez mais se tem procurado aplicar essas técnicas para buscar melhores práticas de manejo de florestas plantadas, seja para fins comerciais ou de recuperação, com a realização de estudos para diferentes espécies nativas da Amazônia (AZEVEDO et al., 1999; TONINI et al., 2005; PEREIRA et al., 2014; SANQUETTA et al., 2015; ALMEIDA et al., 2016; SANQUETTA et al., 2017; CURTO et al., 2018; STOLLE et al., 2018).

Neste contexto, no intuito de qualificar e valorizar espécies amazônicas provenientes de plantios, o objetivo deste trabalho foi avaliar o ajuste de modelos hipsométricos para altura individual total e comercial de árvores de Jatobá em plantios nas idades de 17 e 24 anos, a fim de fornecer estimativas confiáveis para a tomada de decisões referentes a esta espécie no que tange aos serviços ambientais e produção madeireira.

\section{MATERIAL E MÉTODOS}

\section{Área de estudo}

Os dados são oriundos de dois plantios homogêneos da espécie florestal Jatobá (Hymenaea courbaril L.) com 
idades de 17 e 24 anos (P17 e P24, respectivamente). Os plantios estão localizados no Campo Experimental da Embrapa Amazônia Ocidental, o qual se tem acesso pela Rodovia AM 010, Km 29, município de Manaus, estado do Amazonas, Brasil, entre as coordenadas $2^{\circ} 53^{\prime} 30^{\prime \prime} \mathrm{S}$ e $59^{\circ}$ 59' 45" W para P17 e $2^{\circ} 54^{\prime} 04^{\prime \prime}$ S e $59^{\circ} 58^{\prime} 41^{\prime \prime}$ W para P24.

Segundo a classificação de Köeppen, o tipo climático é o Ami, sendo definido por uma estação menos chuvosa, porém com total pluviométrico anual elevado. $\mathrm{A}$ temperatura média anual é de $25,3^{\circ} \mathrm{C}$ com precipitação média de 2.624,2 $\mathrm{mm}$ por ano e umidade relativa média de 86,3\% (ANTÔNIO, 2017). O solo é do tipo Latossolo Amarelo textura argilosa, com baixos valores de $\mathrm{pH}$ e CTC (SOUZA et al., 2010).

Os plantios foram estabelecidos nos anos de 1992 (P24) e 1999 (P17) com o objetivo de avaliar o crescimento sob a condição de pleno sol de espécies florestais com potencialidade para uso como fonte de matéria-prima, em especial para produção de energia e uso em serrarias, e recuperação de áreas degradadas (SOUZA et al., 2008). O plantio P17 foi realizado em dezembro de 1999, em uma parcela com 81 árvores distribuídas em espaçamento retangular de $3 \mathrm{~m} \times 4 \mathrm{~m}$. O plantio P24 foi instalado em janeiro de 1992 em delineamento experimental blocos ao acaso, com quatro repetições de 25 plantas cada, totalizando 100 plantas, em espaçamento $3 \mathrm{~m} \times 3 \mathrm{~m}$.

\section{Coleta de dados}

Foi realizado um inventário $100 \%$ nos dois plantios em março de 2016. Na ocasião, foram coletadas a Circunferência à Altura do Peito (CAP) com fita métrica - a qual posteriormente foi convertida em Diâmetro à Altura do Peito (DAP) -, a altura comercial (HC) e a altura total (HT) das árvores, com a utilização de hipsômetro. A altura comercial foi medida no ponto em que as árvores apresentaram os primeiros galhos.

\section{Ajuste dos modelos e testes de significância}

Para o procedimento de ajuste dos modelos hipsométricos, a base de dados foi dividida em dois grupos, sendo um composto por $70 \%$ dos indivíduos, utilizado para os ajustes dos modelos; outro, com os $30 \%$ restantes, utilizado para a validação das equações obtidas.

Após comprovação de normalidade dos dados e homocedasticidade da variância, foram ajustados 16 modelos hipsométricos (Tabela 1), encontrados na literatura especializada, por meio de regressão linear.

Inicialmente avaliou-se a existência de regressão através do valor de $\mathrm{F}$ resultante da análise de variância. Posteriormente foi feita a análise paramétrica a partir do valor de $t$, atestando significância dos parâmetros estimados, ambos respeitando um nível de significância de $0,05(p<0,05)$.

Tabela 1. Modelos hipsométricos testados para estimativas de altura total e altura comercial em plantios de 17 e 24 anos de Hymenaea courbaril L.

\begin{tabular}{|c|c|c|}
\hline № & Modelo & Identificação \\
\hline 1 & $\hat{h}=\widehat{\beta}_{0}+\widehat{\beta}_{1} \ln (\mathrm{DAP})$ & Henricksen \\
\hline 2 & $\widehat{h}=\hat{\beta}_{0}+\hat{\beta}_{1} \mathrm{DAP}+\hat{\beta}_{2} \mathrm{DAP} \mathrm{P}^{2}$ & Parabólico \\
\hline 3 & $\ln (\hat{h})=\widehat{\beta}_{0}+\widehat{\beta}_{1} 1 / \mathrm{DAP}$ & Curtis \\
\hline 4 & $\ln (\widehat{h})=\widehat{\beta}_{0}+\widehat{\beta}_{1} \ln (\mathrm{DAP})$ & Stofel \\
\hline 5 & $\ln (\hat{h})=\hat{\beta}_{0}+\widehat{\beta}_{1} \ln (\mathrm{DAP})+\widehat{\beta}_{2} 1 / \mathrm{DAP}$ & Silva (1980) \\
\hline 6 & $\widehat{\mathrm{h}}=\widehat{\beta}_{0}+\widehat{\beta}_{1} \mathrm{DAP}$ & Linear \\
\hline 7 & $\widehat{h}=\widehat{\beta}_{0}+\widehat{\beta}_{1} D A P^{2}$ & Azevedo et al. (1999) \\
\hline 8 & $\ln (\hat{h})=\widehat{\beta}_{0}+\widehat{\beta}_{1} D A P^{2}$ & Azevedo et al. (1999) \\
\hline 9 & $\hat{h}=\hat{\beta}_{0}+\hat{\beta}_{1} 1 / \mathrm{DAP}^{2}$ & Soares et al. (2006) \\
\hline 10 & $\widehat{h}=\hat{\beta}_{0}+\hat{\beta}_{1} 1 / \mathrm{DAP}+1 / \mathrm{DAP}^{2}$ & Trorey \\
\hline 11 & $\mathrm{DAP} / \sqrt{\widehat{h}}=\widehat{\beta}_{0}+\widehat{\beta}_{1} \mathrm{DAP}$ & Hiperbólico \\
\hline 12 & $\mathrm{DAP}{ }^{2} / \hat{\mathrm{h}}=\hat{\beta}_{0}+\widehat{\beta}_{1} \mathrm{DAP}+\hat{\beta}_{2} \mathrm{DAP} \mathrm{P}^{2}$ & Prodan \\
\hline 13 & $\mathrm{DAP}^{2} / \widehat{\mathrm{h}}-1,3=\widehat{\beta}_{0}+\widehat{\beta}_{1} \mathrm{DAP}+\widehat{\beta}_{2} \mathrm{DAP} \mathrm{P}^{2}$ & Prodan \\
\hline 14 & $\sqrt{1 / \hat{h}}=\hat{\beta}_{0}+\widehat{\beta}_{1} 1 / \mathrm{DAP}$ & Petterson \\
\hline 15 & $\ln (\widehat{h}-1,3)=\widehat{\beta}_{0}+\widehat{\beta}_{1} 1 / \mathrm{DAP}$ & Pollanschutz \\
\hline 16 & $\mathrm{DAP} / \sqrt{\widehat{h}-1,3=\widehat{\beta}_{0}+\widehat{\beta}_{1} \mathrm{DAP}}$ & Näslund \\
\hline
\end{tabular}

$\hat{\mathrm{h}}=$ altura estimada em metros (total ou comercial); DAP = diâmetro à altura do peito em centímetros; $\hat{\beta}_{\mathrm{n}}=$ parâmetros estimados; In = logaritmo natural.

Atendendo os critérios de significância, a escolha do melhor modelo foi feita considerando, cumulativamente, os seguintes indicadores: maior coeficiente de determinação ajustado ( $R^{2}$ aj.), menor erro padrão da estimativa em porcentagem (Syx\%), o qual foi recalculado para as equações com transformação de variáveis (ver Tabela 1), e a melhor distribuição gráfica dos resíduos.

Após escolha do modelo que melhor representa os dados, a equação foi aplicada no grupo de dados separados para validação (30\% das árvores inventariadas). Nesta etapa foi realizado o teste Qui-Quadrado $(\chi 2)$ ao nível de significância de $0,05(p<0,05)$, onde se testou as 
hipóteses: HO: os valores estimados pelo modelo escolhido são iguais aos valores medidos em campo; $\mathrm{H}$ 1: os valores estimados pelo modelo escolhido são diferentes aos valores medidos em campo.

\section{RESULTADOS E DISCUSSÃO}

A análise descritiva dos dados obtidos após realização dos inventários dos plantios P17 e P24 é apresentada na Tabela 2. Verifica-se que os indivíduos de ambas as idades apresentam coeficientes de variação acima de 35\% para todas as variáveis, sendo que as variáveis que apresentaram menores dispersões foram altura total e comercial para P17. Acredita-se que esta alta variação ocorreu pela não aplicação de técnicas silviculturais que permitissem o desenvolvimento dos povoamentos de forma homogênea. Os tratos aplicados consistiram em capinas e coroamento das plantas a fim de minimizar a competição com as plantas invasoras.

Tabela 2. Estatística descritiva das variáveis dendrométricas nos plantios de Hymenaea courbaril L. aos 17 e 24 anos.

\begin{tabular}{ccccccc}
\hline Idade & NI & Variável & Máx. & Mín. & Média & CV \% \\
\hline \multirow{4}{*}{17 anos } & \multirow{2}{*}{64} & $\operatorname{DAP}(\mathrm{cm})$ & 25,9 & 4,7 & 14,59 & 39,16 \\
& & $\mathrm{HT}(\mathrm{m})$ & 17,5 & 3,0 & 10,92 & 35,78 \\
& & $\mathrm{HC}(\mathrm{m})$ & 9,7 & 1,2 & 5,60 & 35,27 \\
\hline \multirow{4}{*}{24 anos } & \multirow{3}{*}{79} & $\mathrm{DAP}(\mathrm{cm})$ & 45,3 & 6,9 & 25,50 & 37,00 \\
& & $\mathrm{HT}(\mathrm{m})$ & 39 & 4,9 & 18,21 & 43,53 \\
& & $\mathrm{HC}(\mathrm{m})$ & 13,1 & 1,6 & 6,43 & 39,26 \\
\hline
\end{tabular}

$\mathrm{NI}=$ número de indivíduos; $\mathrm{DAP}=$ diâmetro à altura do peito em centímetros; $\mathrm{HT}=$ altura total em metros; $\mathrm{HC}=$ altura comercial em metros; Máx. = valor máximo; Mín. = valor mínimo; $\mathrm{CV}=$ coeficiente de variação.

\section{Ajustes de modelos para estimativa de altura total}

Os resultados da análise de regressão e análise paramétrica para altura total são apresentados na Tabela 3. Todos os modelos testados apresentaram significância ao nível de $1 \%$ de probabilidade para dos dois plantios $(p<0,01)$, indicando que há regressão entre o diâmetro à altura do peito e a altura total das árvores, sendo que os melhores ajustes foram encontrados para P17. Entretanto, a ocorrência de ajustes com parâmetros não significativos indica que nem todas as transformações da variável HT apresentam correlação com o DAP.

Dentre os modelos com o parâmetro $\beta_{2}$, foi observada significância desse parâmetro apenas no ajuste do modelo 10 para P17. Ao analisar a matriz de correlação, verifica-se alta correlação entre as variáveis independentes consideradas nos ajustes ( $r$ entre 0,97 e 0,99), indicando a existência de multicolinearidade. Problema semelhante a este também foi encontrado por Souza et al. (2017) e Stolle et al. (2018) para as variáveis DAP, DAP ${ }^{2}$, InDAP, 1/DAP e 1/DAP ${ }^{2}$. De acordo com Souza et al. (2017), um indício claro da existência da multicolinearidade em regressão é a presença de um alto coeficiente de determinação, mas parâmetros estatisticamente iguais a zero, fato observado nos resultados apresentados na Tabela 3. Para ratificar esta proposição, foi calculado o Fator de Inflação de Variância (FIV) para $\beta_{2}$. Em todos os casos os valores obtidos foram acima de 20, sendo que era esperado valor menor que 10 .

A não significância dos parâmetros $\beta_{2}$ justifica a retirada das variáveis associadas a ele do modelo e novo procedimento de ajuste, o que foi realizado com o modelo 10, gerando o modelo 10a para em P24 (ver Tabela 3). Para os modelos 2 e 5 não foi realizado este procedimento por já haver modelos, dentre os testados, que contemplassem o novo ajuste (modelos 6 e 4, respectivamente). Nos modelos 12 e 13 nenhum parâmetro foi significativo, portanto, não houve novo ajuste.

Em P17, os modelos 11 e 16 apresentaram os maiores $\mathrm{R}^{2}$ aj. - $88 \%$ e $82 \%$, respectivamente - indicando que estes modelos representam mais que $80 \%$ da variabilidade dos dados. Ainda para P17, 11 modelos apresentaram média precisão, com valores de Syx menores que $20 \%$.

No plantio $\mathrm{P} 24$, os valores de $\mathrm{R}^{2} \mathrm{aj}$. indicam que apenas o modelo 11 consegue descrever mais que $60 \%$ da variabilidade dos dados. Todos os modelos podem ser considerados com baixa precisão, apresentando Syx próximos a 30\%, sendo no caso dos modelos 7, 8 e 9, Syx maior que $30 \%$. Com parâmetros não significativos, os modelos 11 e 12 foram casos extremos de erro de estimativa.

A análise gráfica dos resíduos foi realizada apenas para os modelos que apresentaram parâmetros significativos, conforme Tabela 3. Todos os modelos apresentaram distribuição semelhante, nas quais foi identificada uma maior tendência a erros relacionados a superestimativas da altura total nas duas idades.

Para P17 os erros máximos de subestimativa ficaram abaixo de 40\%, com exceção do modelo 9, que apresentou erro máximo de $85 \%$; quanto aos erros de superestimativas, os maiores valores observados ficaram entre $65 \%$ e $100 \%$ de erro. Para P24, as superestimativas apresentaram erros máximos entre $40 \%$ e 50\%, com exceção do modelo 9, com valor de $67 \%$. Para erros de 
subestimativas, foram encontrados os valores maiores, variando de $200 \%$ a $250 \%$, com exceção do modelo 8 , com erro máximo de $181 \%$. Na Figura 1 são apresentados os gráficos de dispersão de resíduos do modelo 11 para P17 (Figura 1a) e P24 (Figura 1b), o qual apresentou o melhor ajuste dentre os 16 testados. Em P24 é observado um outlier, o qual representa uma árvore com DAP igual a 19,9 cm e HT de 4,9 m, sendo que os demais indivíduos com diâmetro entre 19 e $20 \mathrm{~cm}$ apresentam HT acima de $15 \mathrm{~m}$.

Tabela 3. Parâmetros estimados e estatística de precisão dos modelos hipsométricos ajustados para estimativa de altura total em plantios de Hymenaea courbaril L. aos 17 e 24 anos. Em destaque o modelo escolhido com melhor desempenho.

\begin{tabular}{|c|c|c|c|c|c|c|c|c|}
\hline \multirow{2}{*}{ Idade } & \multirow{2}{*}{ № } & \multirow{2}{*}{$\mathbf{F}$} & \multicolumn{3}{|c|}{ Parâmetros } & \multirow{2}{*}{$R^{2}$ aj. (\%) } & \multirow{2}{*}{$r$} & \multirow{2}{*}{$S_{y x}(\%)$} \\
\hline & & & $\beta_{0}$ & $\beta_{1}$ & $\beta_{2}$ & & & \\
\hline \multirow{16}{*}{$\begin{array}{c}17 \\
\text { anos }\end{array}$} & 1 & $119,59^{* *}$ & $-8,506857$ & $7,617586^{* *}$ & & 73,55 & 0,86 & 17,04 \\
\hline & 2 & $61,67^{* *}$ & 0,535457 & $0,934532^{* *}$ & $-0,012003^{\text {ns }}$ & 74,60 & 0,86 & 16,90 \\
\hline & 3 & $111,34^{* *}$ & 3,040759 & $-8,262471^{* *}$ & & 72,14 & 0,85 & 17,65 \\
\hline & 4 & $133,19^{* *}$ & 0,407431 & $0,750012^{* *}$ & & 75,59 & 0,87 & 16,89 \\
\hline & 5 & $65,05^{* *}$ & 0,344777 & $0,767656^{*}$ & $0,203470^{\text {ns }}$ & 75,60 & 0,87 & 17,10 \\
\hline & 6 & $120,52^{* *}$ & 2,876517 & $0,572227^{* *}$ & & 73,70 & 0,86 & 17,00 \\
\hline & 7 & $94,90^{* *}$ & 6,822214 & $0,018047^{* *}$ & & 68,82 & 0,83 & 18,51 \\
\hline & 8 & $79,30^{* *}$ & 1,937295 & $0,001701^{* *}$ & & 64,84 & 0,81 & 21,17 \\
\hline & 9 & $53,88^{* *}$ & 14,381481 & $-354,926202^{* *}$ & & 55,61 & 0,75 & 22,08 \\
\hline & 10 & $60,28^{* *}$ & 23,501895 & $-213,017056^{* *}$ & $636,247398^{* *}$ & 74,16 & 0,86 & 17,05 \\
\hline & 11 & $310,40^{* *}$ & 1,519859 & $0,189767^{* *}$ & & 87,83 & 0,94 & 16,96 \\
\hline & 12 & $123,30^{* *}$ & 0,192720 & $0,952023^{\text {ns }}$ & $0,023052^{\text {ns }}$ & 85,45 & 0,92 & 34,31 \\
\hline & 13 & $77,71^{* *}$ & 1,970868 & $1,016229^{n s}$ & $0,022936^{n s}$ & 78,73 & 0,89 & 44,80 \\
\hline & 14 & $115,93^{* *}$ & 0,201363 & $1,357152^{* *}$ & & 72,94 & 0,85 & 17,35 \\
\hline & 15 & $113,27^{* *}$ & 3,012456 & $-9,673539^{* *}$ & & 72,48 & 0,85 & 30,34 \\
\hline & 16 & $191,34^{* *}$ & 1,902062 & $0,184173^{* *}$ & & 81,65 & 0,90 & 16,92 \\
\hline \multirow{17}{*}{$\begin{array}{c}24 \\
\text { anos }\end{array}$} & 1 & $57,96^{* *}$ & $-24,608696$ & $13,709058^{* *}$ & & 52,23 & 0,72 & 28,28 \\
\hline & 2 & $28,52^{* *}$ & $-2,502345$ & $1,139990^{* *}$ & $-0,010560^{\text {ns }}$ & 52,31 & 0,72 & 28,53 \\
\hline & 3 & $65,96^{* *}$ & 3,591868 & $-16,416587^{* *}$ & & 55,45 & 0,74 & 28,68 \\
\hline & 4 & $67,13^{* *}$ & 0,073685 & $0,878424^{* *}$ & & 55,88 & 0,75 & 28,80 \\
\hline & 5 & $33,24^{* *}$ & 1,370664 & $0,556013^{\text {ns }}$ & $-6,141080^{\text {ns }}$ & 56,11 & 0,75 & 28,86 \\
\hline & 6 & $55,07^{* *}$ & 2,856441 & $0,635052^{* *}$ & & 50,96 & 0,71 & 28,66 \\
\hline & 7 & $44,60^{* *}$ & 10,182115 & $0,012320^{* *}$ & & 45,69 & 0,68 & 30,15 \\
\hline & 8 & $42,53^{* *}$ & 2,324932 & $0,000753^{* *}$ & & 44,52 & 0,67 & 34,49 \\
\hline & 9 & $43,72^{* *}$ & 23,347157 & $-2005,024171^{* *}$ & & 45,20 & 0,67 & 30,29 \\
\hline & 10 & $28,75^{* *}$ & 39,514818 & $-625,464326^{* *}$ & $3173,784457^{\text {ns }}$ & 52,51 & 0,72 & 28,47 \\
\hline & $10 a$ & $52,64^{* *}$ & 30,052404 & $-251,219286^{* *}$ & & 49,83 & 0,71 & 28,98 \\
\hline & 11 & $86,03^{* *}$ & 2,277551 & $0,143061^{* *}$ & & 61,88 & 0,79 & 28,90 \\
\hline & 12 & $31,30^{* *}$ & 3,219191 & $0,894311^{\text {ns }}$ & $0,015717^{\text {ns }}$ & 54,62 & 0,74 & 69,96 \\
\hline & 13 & $18,85^{* *}$ & 6,110467 & $0,926977^{n s}$ & $0,015272^{\text {ns }}$ & 42,04 & 0,65 & 90,74 \\
\hline & 14 & $60,35^{* *}$ & 0,146507 & $2,203585^{* *}$ & & 53,24 & 0,73 & 29,02 \\
\hline & 15 & $64,43^{* *}$ & 3,585018 & $-18,204255^{* *}$ & & 54,87 & 0,74 & 28,71 \\
\hline & 16 & $55,37^{* *}$ & 2,670180 & $0,137858^{* *}$ & & 51,09 & 0,71 & 29,09 \\
\hline
\end{tabular}

ns = valor não significativo; * = significativo ao nível de $5 \%$ de probabilidade; ${ }^{* *}=$ significativo ao nível de $1 \%$ de probabilidade

Aplicando-se a equação ajustada selecionada para estimativa de altura total na base de dados separada para validação, foi constatada diferença significativa para P24 $\left(p=0,11 \times 10^{-12}\right)$. Dessa forma, deve-se rejeitar $\mathrm{HO}$ e assumir a proposição de $\mathrm{H} 1$, em que há diferença entre os valores observados e os estimados pela equação. Voltando à Tabela 2, é visto que este plantio é o que apresenta maior coeficiente de variação para altura total 
$(43,5 \%)$, tornando mais difícil uma modelagem hipsométrica mais precisa. Para $\mathrm{P} 17$, não foi observada diferença significativa $(p=0,99)$, dessa forma aceita-se a proposição de $\mathrm{HO}$.

\section{Ajustes de modelos para estimativa de altura comercial}

Os resultados obtidos nos ajustes dos modelos hipsométricos para altura comercial são apresentados na Tabela 4. Apenas o modelo 16 não apresentou regressão; todos os outros alcançaram significância ao nível de $1 \%$ de

a)

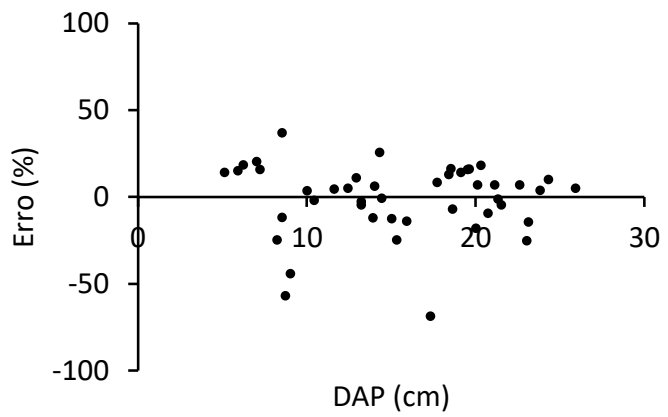

c)

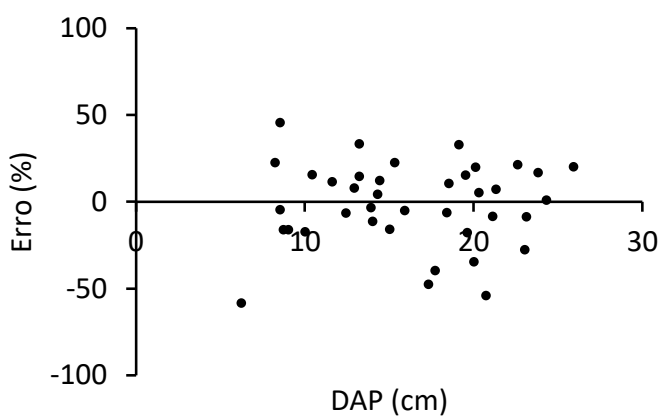

probabilidade $(p<0,01)$.

Nenhum modelo de dupla entrada apresentou significância para o parâmetro $\beta_{2}$, sendo que em alguns casos ambos os parâmetros foram não significativos. Da mesma forma, como observado nos ajustes para altura total, algumas transformações dos valores de DAP não apresentam correlação significativa com a altura comercial. Para o modelo 10 foi realizado o mesmo procedimento de retirada do parâmetro não significativo, gerando o modelo 10a.

b)

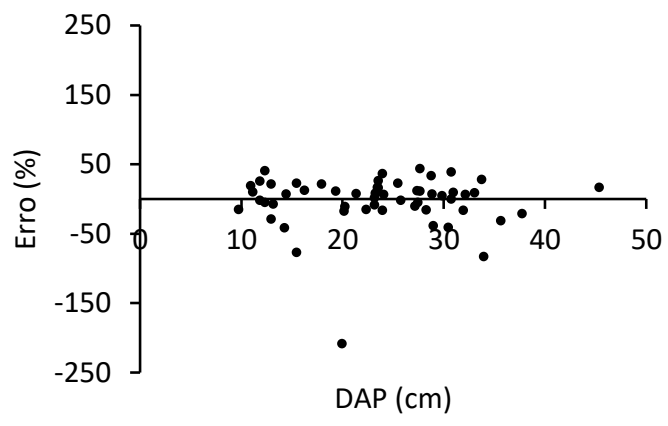

d)

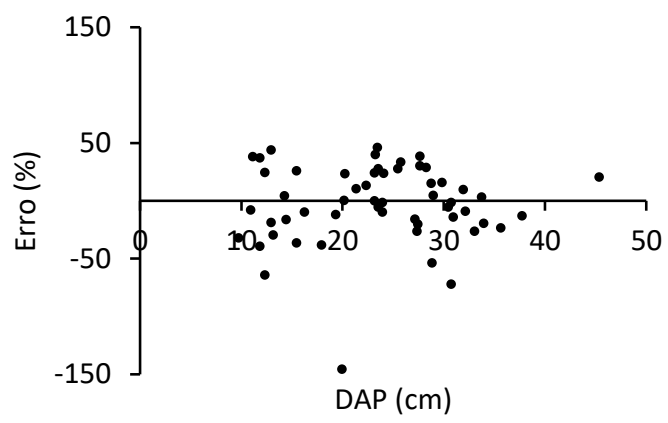

Figura 1. Gráficos de dispersão de resíduos do modelo 11 para estimativas de altura total e comercial de árvores de Hymenaea courbaril L. em plantios homogêneos aos 17 e 24 anos de idade: a) Altura total aos 17 anos; b) Altura total aos 24 anos; c) Altura comercial aos 17 anos; d) Altura comercial aos 24 anos.

Em cada plantio, apenas dois modelos conseguiram um desempenho razoável na representatividade dos dados, com $\mathrm{R}^{2}$ aj. acima de $70 \%$. Em P17, os modelos $11 \mathrm{e}$ 14 apresentaram $\mathrm{R}^{2}$ aj. de $72 \%$ e $71 \%$, respectivamente. Em P24, os melhores desempenhos foram dos modelos 11 e 12, entretanto, este último apresentou parâmetros não significativos. Todos os valores de Syx foram maiores que $20 \%$, indicando uma baixa precisão nas estimativas.

Como observado nas estimativas de altura total, os gráficos de dispersão de resíduos para altura comercial se apresentaram semelhantes entre si e com maior tendência a erros de superestimativas. Em P17, a maioria dos modelos apresentou erros máximos de superestimativas entre $50 \%$ e $60 \%$; quatro modelos destoaram desse padrão: modelo 4 (80\%), modelo 6 (108\%), modelo 7 (167\%) e modelo 13 (1768\%). Quanto aos erros de subestimativas, os valores máximos variaram de $39 \%$ a 48\%, com exceção dos modelos 9 e 13, cujos erros máximos foram de $116 \%$ e $208 \%$, respectivamente. Para P24, a variação dos erros máximos de superestimativas ficou entre $134 \%$ e $178 \%$; já os de subestimativas ficaram entre $40 \%$ e $50 \%$. Na Figura 1 são apresentados os gráficos de dispersão de resíduos do modelo 11, o qual apresentou o melhor ajuste dentre os 16 testados, para P17 (Figura 1c) e P24 (Figura 1d).

A validação dos resultados por meio do teste Qui- 
Quadrado não indicou diferença significativa tanto para P17 ( $p=0,45)$, quanto para P24 $(p=0,58)$. Dessa forma, aceita-se H0, em que altura comercial estimada é estatisticamente igual à altura comercial observada em campo.

De forma geral, o melhor ajuste foi obtido para altura total no plantio de 17 anos, no qual foi alcançado um coeficiente de determinação ajustado de quase 90\%. Para altura comercial, os melhores ajustes indicaram uma representatividade de pouco mais de $70 \%$, o suficiente para ser aceita a hipótese de igualdade estatística entre os valores reais e estimados. Dentre os modelos selecionados após análises, o de menor representatividade foi o de P24 para altura total. Acredita-se que a variação de mais de $40 \%$ nos dados de inventário seja responsável por esse resultado, uma vez que dificulta a determinação de parâmetros abrangentes a esta variação.

Tabela 4. Parâmetros estimados e estatística de precisão dos modelos hipsométricos ajustados para estimativa de altura comercial em plantios de Hymenaea courbaril L. aos 17 e 24 anos. Em destaque o modelo escolhido com melhor desempenho.

\begin{tabular}{|c|c|c|c|c|c|c|c|c|}
\hline \multirow{2}{*}{ Idade } & \multirow{2}{*}{ № } & \multirow{2}{*}{$\mathbf{F}$} & \multicolumn{3}{|c|}{ Parâmetros } & \multirow{2}{*}{$R^{2}$ aj. (\%) } & \multirow{2}{*}{$r$} & \multirow{2}{*}{$\mathrm{S}_{\mathrm{yx}}(\%)$} \\
\hline & & & $\beta_{0}$ & $\beta_{1}$ & $\beta_{2}$ & & & \\
\hline \multirow{17}{*}{$\begin{array}{c}17 \\
\text { anos }\end{array}$} & 1 & $58,12^{* *}$ & $-6,229864$ & $2,147455^{* *}$ & & 61,10 & 0,78 & 23,06 \\
\hline & 2 & $28,98^{* *}$ & 0,491385 & $0,321113^{\text {ns }}$ & $-0,000750^{\text {ns }}$ & 61,68 & 0,79 & 23,21 \\
\hline & 3 & $85,64^{* *}$ & 2,493019 & $-12378603^{* *}$ & & 69,83 & 0,83 & 23,36 \\
\hline & 4 & $73,85^{* *}$ & $-0,952506$ & $0,472732^{* *}$ & & 66,62 & 0,82 & 83,21 \\
\hline & 5 & $41,76^{* *}$ & 2,971094 & $-0,066280^{\text {ns }}$ & $-14,042790^{\text {ns }}$ & 69,88 & 0,84 & 30,88 \\
\hline & 6 & $59,54^{* *}$ & 0,662172 & $0,297160^{* *}$ & & 61,67 & 0,79 & 22,89 \\
\hline & 7 & $55,35^{* *}$ & 2,851652 & $0,009062^{* *}$ & & 59,94 & 0,77 & 23,41 \\
\hline & 8 & $44,72^{* *}$ & 1,095723 & $0,001826^{* *}$ & & 54,72 & 0,74 & 24,71 \\
\hline & 9 & $38,13^{* *}$ & 7,071593 & $-279,267680^{* *}$ & & 50,75 & 0,71 & 25,95 \\
\hline & 10 & $26,86^{* *}$ & 11,328614 & $-109,422839^{* *}$ & $319,615216^{\mathrm{ns}}$ & 59,88 & 0,77 & 23,75 \\
\hline & $10 a$ & $49,81^{* *}$ & 9,210917 & $-53,223576^{* *}$ & & 57,38 & 0,76 & 24,14 \\
\hline & 11 & $95,30^{* *}$ & 2,982621 & $0,244481^{* *}$ & & 72,03 & 0,85 & 23,31 \\
\hline & 12 & $39,31^{* *}$ & $-2,384193$ & $3,124667^{\text {ns }}$ & $0,007693^{\text {ns }}$ & 68,59 & 0,83 & 225,96 \\
\hline & 13 & $11,93^{* *}$ & $-312,671059$ & $42,403693^{* *}$ & $-1,094746^{* *}$ & 39,86 & 0,63 & 1127,66 \\
\hline & 14 & $91,56^{* *}$ & 0,226664 & $3,245053^{* *}$ & & 71,22 & 0,84 & 23,28 \\
\hline & 15 & $55,57^{* *}$ & 2,416197 & $-15,761155^{* *}$ & & 60,68 & 0,78 & 23,15 \\
\hline & 16 & $1,38^{\text {ns }}$ & 6,998395 & $0,088339^{\text {ns }}$ & & 3,60 & 0,19 & 24,89 \\
\hline \multirow{16}{*}{$\begin{array}{c}24 \\
\text { anos }\end{array}$} & 1 & $17,01^{* *}$ & $-2,507925$ & $3,020840^{* *}$ & & 24,29 & 0,49 & 29,87 \\
\hline & 2 & $8,52^{* *}$ & 0,942094 & $0,394316^{* *}$ & $-0,005480^{\text {ns }}$ & 24,67 & 0,50 & 30,09 \\
\hline & 3 & $23,35^{* *}$ & 2,357028 & $-10,183573^{* *}$ & & 30,58 & 0,55 & 30,01 \\
\hline & 4 & $21,44^{* *}$ & 0,230663 & $0,526746^{* *}$ & & 28,80 & 0,54 & 30,54 \\
\hline & 5 & $11,57^{* *}$ & 3,417837 & $-0,265542^{n s}$ & $-15,090979^{n s}$ & 30,80 & 0,55 & 30,16 \\
\hline & 6 & $14,24^{* *}$ & 3,722987 & $0,132282^{* *}$ & & 21,18 & 0,46 & 30,48 \\
\hline & 7 & $10,92^{* *}$ & 5,329569 & $0,002434^{* *}$ & & 17,09 & 0,41 & 31,26 \\
\hline & 8 & $13,24^{* *}$ & 1,599031 & $0,000422^{* *}$ & & 19,98 & 0,45 & 32,31 \\
\hline & 9 & $17,97^{* *}$ & 8,180592 & $-484,859024^{* *}$ & & 25,32 & 0,50 & 29,67 \\
\hline & 10 & $8,99^{* *}$ & 9,365854 & $-45,853213^{n s}$ & $-105,197061^{\text {ns }}$ & 25,70 & 0,51 & 29,88 \\
\hline & 11 & $165,02^{* *}$ & 2,031183 & $0,301745^{* *}$ & & 75,69 & 0,87 & 29,73 \\
\hline & 12 & $69,24^{* *}$ & $-2,970089$ & $1,992162^{\text {ns }}$ & $0,076384^{\mathrm{ns}}$ & 72,70 & 0,85 & 420,80 \\
\hline & 13 & $25,86^{* *}$ & 9,163886 & $2,487955^{\text {ns }}$ & $0,081282^{\text {ns }}$ & 49,86 & 0,71 & 776,87 \\
\hline & 14 & $24,70^{* *}$ & 0,293458 & $2,212902^{* *}$ & & 31,79 & 0,56 & 30,51 \\
\hline & 15 & $24,01^{* *}$ & 2,279537 & $-13,775894^{* *}$ & & 31,17 & 0,56 & 30,30 \\
\hline & 16 & $67,65^{* *}$ & 3,279068 & $0,301373^{* *}$ & & 56,07 & 0,75 & 31,18 \\
\hline
\end{tabular}

ns = valor não significativo; ${ }^{*}=$ significativo ao nível de $5 \%$ de probabilidade; ${ }^{* *}=$ significativo ao nível de $1 \%$ de probabilidade. 
De acordo com Campos; Leite (2006), é comum que em relações hipsométricas os valores de $R^{2}$ aj. fiquem abaixo de $80 \%$, uma vez que a relação entre altura e diâmetro é menos forte que a relação entre altura e volume. Stolle et al. (2018) encontraram $R^{2}$ aj abaixo de $50 \%$ para ajustes hipsométricos para plantios de Mogno Africano. Em ajustes para Acácia-negra, Sanqueta et al. (2014) encontraram $R^{2}$ aj entre 35 e 63\%. Em plantio de Teca, Pereira et al. (2014), considerando os mesmos modelos aplicados no presente estudo, encontraram coeficientes de determinação entre $45 \%$ e $47 \%$; para a mesma espécie Sanquetta et al. (2015) obtiveram $R^{2} a j$ próximos a $90 \%$. Considerando plantios de quatro espécies nativas (sete-cascas, ipê branco, ipê roxo e ipê amarelo), Azevedo et al. (2011) encontraram nos melhores ajustes $\mathrm{R}^{2}$ aj entre $67 \%$ e $99 \%$.

\section{CONLUSÕES}

A modelagem hipsométrica em plantios de Jatobá aos 17 e 24 anos de idade obteve igualdade estatística entre os dados estimados e observados para as variáveis com CV menor que $40 \%$.

O modelo Hiperbólico se mostrou o mais apropriado para estimativas tanto de altura total, quanto de altura comercial.

Apesar de boa representatividade dos dados, pode-se considerar a modelagem hipsométrica dos dados de média à baixa precisão, uma vez que os erros padrão ficaram entre $17 \%$ e $30 \%$.

\section{REFERÊNCIAS}

ALMEIDA, D.L.C.S. et al. Determinação de equação volumétrica e hipsométrica para um plantio de Tectona grandis L. f. em Alta Floresta, MT. Revista de Ciências Agroambientais, v.14, n.2, p.19, 2016.

ALVES, D.S. et al. Taxas de mudança e padrões de desmatamento e uso da terra na Amazônia brasileira. Washington: American Geophysical Union, 2009.

ANTONIO, I.C. Boletim agrometeorológico série anual: 2016: Estação Agroclimatológica da Embrapa Amazônia Ocidental na Rodovia AM-010, Km 29. Manaus: Embrapa Amazônia Ocidental, 2017.

AZEVEDO, C.P. et al. Relação hipsométrica para quatro espécies florestais em plantio homogêneo e em diferentes idades na Amazônia ocidental. Boletim de Pesquisa Florestal, n.39, p.5-29, 1999.

AZEVEDO, G.B. et al. Seleção de modelos hipsométricos para quatro espécies florestais nativas em plantio misto no planalto da conquista na Bahia. Enciclopédia Biosfera, v.7, n.12, p.1-13,
2011.

BRIENZA JÚNIOR, S. et al. Recuperação de áreas degradadas com base em sistema de produção florestal energético-madeireiro: indicadores de custos, produtividade e renda. Amazônia: Ciência \& Desenvolvimento, v.4, n.7, p.197-219, 2008.

CAMPOS FILHO, EM.; SARTORELLI, P.A. R. Guia de árvores com valor econômico. São Paulo: Agroícone, 2015.

CAMPOS, J.C.C.; LEITE, H.G. Mensuração florestal: perguntas e respostas. 2 ed. Viçosa: UFV, 2006.

CAMPOS, M.A.A.; UCHIDA, T. Influência do sombreamento no crescimento de mudas de três espécies amazônicas. Pesquisa Agropecuária Brasileira, v.37, n.3, p.281-288, 2002.

CURTO, R.A. et al. Modelos de relação hipsométrica por classe de aproveitamento em floresta de transição cerrado-amazônia no Mato Grosso. Enciclopédia Biosfera, v.15, n.28, p.687-700, 2018.

FELICIANO, M.E. et al. Avaliação de diferentes hipsômetros na estimativa da altura total. Revista Verde de Agroecologia e Desenvolvimento Sustentável, v.11, n.2, p.1-5, 2016.

KELLER, M. et al. The large-scale biosphere-atmosphere experiment in Amazonia: analyzing regional land use change effects. Ecosystems and Land Use Change. Boston: American Geophysical Union, 2004.

LORENZI, H. Árvores brasileiras: manual de identificação e cultivo de plantas arbóreas nativas do Brasil. Nova Odessa: Instituto Plantarum, v.2, 2002.

MACHADO, S.A.; FIGUEIREDO FILHO, A. Dendrometria. Curitiba: Editado pelos autores, 2003.

MELO, M.G.G. et al. Análise morfológica de sementes, germinação e plântulas de Jatobá (Hymenaea intermedia Ducke var. adenotricha (Ducke) Lee \& Lang.) (Leguminosaecaesalpinioideae). Acta Amazonica, v.34, n.1, p.9-14, 2004.

MIRANDA, E.M.; VALENTIM, J.F. Desempenho de doze espécies arbóreas nativas e introduzidas com potencial de uso múltiplo no estado do Acre, Brasil. Acta Amazonica, v.30, n.3, p.471-480, 2000.

MONTEIRO, A.L.S. et al. Impactos da exploração madeireira e do fogo em florestas de transição da Amazônia Legal. Scientia Forestalis, n.65, p.11-21, 2004.

NASCIMENTO, H.H.C. et al. Análise do Crescimento de mudas de Jatobá (Hymenaea courbaril L.) em diferentes níveis de água no solo. Revista Árvore, v.35, n.3, p.617-626, 2011.

PEREIRA, K.D. et al. Ajuste de modelos hipsométricos para árvores de Tectona grandis L.f. no município de Mojú, Pará. Enciclopédia Biosfera, v.10, n.18, p.181-189, 2014.

SANQUETTA, C.R. et al. Inventários Florestais: planejamento e execução. 3.ed. Curitiba: Multi-Graphic Gráfica e Editora, 2014.

SANQUETTA, C.R. et al. Estimativa da altura e do volume em 
povoamentos jovens de restauração florestal em Rondônia. Biofix Scientific Journal, v.2, n.2, p.23-31, 2017.

SANQUETTA, C.R. et al. Relação hipsométrica em inventários pré-corte em povoamentos de Acacia mearnsii De Wild. Científica, v.42, n.1, p.80-90, 2014.

SANQUETTA, M.N.I. et al. Ajuste de equações hipsométricas para a estimação da altura total de indivíduos jovens de teca. Científica, v.43, n.4, p.400-406, 2014.

SCHNEIDER, P.R. et al. Análise de regressão aplicada a Engenharia Florestal. 2.ed. Santa Maria: FACOS-UFSM, 2009.

SILVA, J.NM. Relação hipsométrica de espécies da Floresta Nacional do Tapajós. Belém: EMBRAPA-CPATU, 1980.

SILVA, G.F. et al. Simulação de erros na medição de altura de árvores inclinadas com aparelhos baseados em princípios trigonométricos. Revista Nativa, v.5, n.5, p.372-379, 2017.

SOARES, C.P.B. et al. Dendrometria e Inventário Florestal. Viçosa: UFV, 2006.

SOUZA, A.S. et al. Modelagem da relação hipsométrica para um povoamento híbrido de eucalipto na Amazônia brasileira. Biofix Scientific Journal, v.2, n.1, p.44-53, 2017.

SOUZA, C.R. et al. Desempenho de espécies florestais para uso múltiplo na Amazônia. Scientia Forestalis, v.36, n.77, p.7-14, 2008.

SOUZA, C.R. et al. Comportamento de espécies florestais em plantios a pleno sol e em faixas de enriquecimento de capoeira na Amazônia. Acta Amazonica, v.40, n.1, p.127-134,2010.

STOLLE, L. et al. Modelos hipsométricos para um povoamento jovem de Khaya ivorensis A. Chev. Biofix Scientific Journal, v.3, n.2, p.231-236, 2018.

TONINI, H. et al. Dendrometria de espécies nativas em plantios homogêneos no Estado de Roraima - Andiroba (Carapa guianensis Aubl), Castanha-do-Brasil (Bertholletia excelsa Bonpl.), Ipê-roxo (Tabebuia avellanedae Lorentz ex Griseb) e Jatobá (Hymenaea courbaril L.). Acta Amazonica, v.35, n.3, p.353-362, 2005.

Recebido em 25-11-2019 Aceito em 27-01-2020 